\title{
Desarrollo de la fuerza en el adulto mayor a través de la hidrogimnásia en la calidad de vida
}

\author{
DOI: https://doi.org/10.33262/ap.v2i3.35
}

\begin{abstract}
(c) (1) (8)(2)
\end{abstract}
Development of strength in the elderly adult through hydrogymnasis in quality of life

\begin{abstract}
Mónica Cabezas Cabezas Flores., ${ }^{1}$ Orlando Rodrigo Carrasco Coca., ${ }^{2}$ Vanessa Lucia Ochoa Sangurima., ${ }^{3}$ \& Ruth Sayonara Ríos Bayas. ${ }^{4}$
\end{abstract}

\begin{abstract}
.
The practice of regular physical activity is one of the priorities in public health as a form of prevention of chronic degenerative diseases, especially in the Third Age. The main scientifically proven benefits are: control of body weight, decrease in body fat, increase in muscle mass, muscle strength, flexibility and bone density, increase in stroke volume, pulmonary ventilation, maximum oxygen consumption, decrease in heart rate and blood pressure and lipid profile improvement; There is also an improvement in self-concept, selfesteem, body image and a decrease in stress, anxiety, insomnia, drug use, and an improvement in cognitive functions and socialization. Low-impact aerobic activities such as walking, swimming, dancing, hydrorogimnásia or cycling are preferably indicated at this stage of life. Strength training is essential and can be prescribed with some care even in hypertensive individuals or with cardiovascular problems. Training contributes to improve

${ }^{1}$ Universidad de las Fuerzas Armadas -“ESPE”, Carrera de Pedagogía de la Actividad Física y Deportes. Quito, Ecuador.mmcabezas@espe.edu.ec

${ }^{2}$ Universidad de las Fuerzas Armadas -“ESPE”, Carrera de Pedagogía de la Actividad Física y Deportes. Quito, Ecuador. orcarrasco@espe.edu.ec

${ }^{3}$ Universidad de las Fuerzas Armadas -“ESPE”, Carrera de Pedagogía de la Actividad Física y Deportes. Quito, Ecuador.vane4lali@gmail.com

${ }^{4}$ Universidad de las Fuerzas Armadas -"ESPE”, Carrera de Pedagogía de la Actividad Física y Deportes. Quito, Ecuador.rsrios@espe.edu.ec
\end{abstract}


muscle strength, to maintain functional independence, improves walking speed and balance, reduces depression, increases bone density and prevents falls. There are some specific clinical situations in which muscle strength training should be a priority over aerobic training, such as severe arthritis, limited joint mobility, amputation, and patients with locomotion problems. To maintain functional independence, the priority of variables that must be worked on are: muscle strength training, balance, aerobic training, total body movements.

Keywords: Hydrogymnastics, strength, older adult, quality of life, physical activity.

\section{Resumen.}

La práctica de actividad física regular es una de las prioridades en salud pública como forma de prevención de enfermedades crónico-degenerativas especialmente en la Tercera Edad. Los principales beneficios evidenciados científicamente son: control del peso corporal, disminución de la grasa corporal, aumento de la masa muscular, fuerza muscular, flexibilidad y densidad ósea, aumento del volumen sistólico, ventilación pulmonar, consumo máximo de oxígeno, disminución de la frecuencia cardíaca y de la presión arterial y mejora del perfil de lípidos; existe también mejora del auto-concepto, auto-estima, imagen corporal y disminución del stress, ansiedad, insomnio, consumo de medicamentos y mejora de las funciones cognitivas y de la socialización. En esta etapa de la vida están indicadas de preferencia las actividades aeróbicas de bajo impacto, como caminar, nadar, bailar, la hidrogimnásia o bicicleta. El entrenamiento de fuerza es fundamental y puede ser prescrito con algunos cuidados inclusive en individuos hipertensos o con problemas cardiovasculares. El en entrenamiento contribuye para mejorar la fuerza muscular, para la manutención de la independencia funcional, mejora la velocidad de andar y el equilibrio, disminuye la depresión, aumenta la densidad ósea y evita las caídas. Existen algunas situaciones clínicas específicas en que el entrenamiento de fuerza muscular debe ser prioritario al entrenamiento aeróbico como en los casos de artritis severa, limitación de la movilidad articular, amputación y pacientes con problemas de locomoción. Para mantener la independencia funcional la prioridad de variables que deben ser trabajadas son: entrenamiento de la fuerza muscular, equilibrio, entrenamiento aeróbico, movimientos corporales totales.

Palabras claves: Hidrogimnásia, fuerza, adulto mayor, calidad de vida, actividad física.

\section{Introducción.}

Una de las principales preocupaciones de los científicos es con el envejecimiento de nuestras poblaciones. Sabemos que tanto en los países desarrollados como en los que están en vía de desarrollo existe una tendencia a aumentar la expectativa de vida alnacer y a que tengamos cada vez más personas mayores de 60 años de edad. Uno de los factores determinantes de los efectos deletéreos del envejecimiento es la disminución del nivel de actividad física. A medida que envejecemos nos volvemos más sedentarios y este menor 
nivel de actividad hace con que perdamos capacidades y habilidades físicas. La principal estrategia para disminuir los efectos del envejecimiento esta por tanto en evitar la pérdida de actividad física que ocurre con la edad. Sabemos también que el envejecimiento está acompañado por una serie de alteraciones en todos los sistemas del organismo que afectan de forma importante la aptitud física. Informaciones recientes de nuestro Centro de Investigaciones (Andrade, 2014) en mujeres practicantes de actividad física de 30 a 73 años de edad evidenciaron que existe un declinó del desempeño neuromotor con los años, siendo que esta pérdida es mayor en las variables de agilidad y fuerza de los miembros inferiores de lo que para la fuerza de los miembros superiores. Considerando los datos citados por los autores la pérdida para estas variables de los 30 a los 73 años es de: $67 \%$ para la agilidad; $58 \%$ para la fuerza de los miembros inferiores y $28 \%$ para la fuerza de los miembros superiores. Analizando el desempeño neuromotor en relación al índice de masa corporal (Andrade, 2014) fue observado que de acuerdo al grado de obesidad los resultados de los testes neuromotores, especialmente para agilidad y fuerza de los miembros inferiores, eran peores en las mujeres obesas. Las investigaciones científicas muestran también que la pérdida de fuerza muscular es de aproximadamente $30 \%$ y del área muscular de $40 \%$ de la $2^{\mathrm{a}}$ a $7^{\mathrm{a}}$ décadas de la vida. Esta pérdida ocurre básicamente por la disminución en el número y tamaño de las fibras musculares tipo I y tipo II, siendo que existe una perdida diferenciada dependiendo del tipo de fibra muscular. Con el envejecimiento son perdidas más fibras de tipo II que de tipo I y son perdidas más del tipo II b que del tipo II a. Estas alteraciones comprometen significativamente la capacidad funcional del anciano principalmente todos los aspectos relacionados a velocidad, agilidad y tiempo de reacción.

\section{Actividad física para el adulto mayor.}

La promoción de actividad física en los adultos mayores es indispensable para disminuir los efectos del envejecimiento y preservar la capacidad funcional del anciano. Existen varios tipos de actividades que pueden y deben ser promovidos en esta edad. Algunos de ellos son:

Actividades Aeróbicas: es recomendada la realización de actividades de bajo impacto como la caminada, el ciclismo o pedalear en la bicicleta, la natación, la hidrogimnásia, subir escaleras, bailar, yoga, tai chi chuan y gimnasia aeróbica de bajo impacto. Estas actividades son preferibles a las llamadas de alto impacto, como trotar, correr, o practicar deportes con saltos, como el volibol o basquetbol y gimnasia aeróbica de alta impacto, que tienen grande prevalencia de lesiones en esta época de la vida.

Entrenamiento de la Fuerza Muscular: un aspecto fundamental del programa de ejercicio es el fortalecimiento de la musculatura buscando aumentar la masa muscular y la fuerza muscular; evitando así una de las principales causas de incapacidad y de caídas. Además la masa muscular es el principal estímulo para aumentar la densidad ósea.

En un trabajo clásico realizado en individuos de 60-96 años que participaron de un programa 
de entrenamiento de 8 semanas (3 veces/semana) para fortalecer la musculatura de los miembros inferiores mostro mejora de $174 \%$ en la fuerza y $48 \%$ en la velocidad de andar. Sin embargo, 4 semanas de suspensión del entrenamiento provocaron una disminución de $32 \%$ en la fuerza. Continuando en esta línea de investigación varios trabajos científicos han sido realizados en CELAFISCS en los últimos años (Raso, 2014) propusieron tres protocolos distintos de ejercicios físicos entrenamiento de fuerza muscular, aeróbico y una asociación del entrenamiento de fuerza muscular y el aeróbico para verificar los efectos sobre el peso, la adiposidad y el índice de masa corporal en mujeres saludables de 55 a 80 años de edad durante 4 semanas. A pesar de verificar que el corto período de tiempo no fue suficiente para promover alteraciones significativas en las variables antropométricas, fue observado que el grupo que realizó ejercicios de fuerza muscular, disminuyó la adiposidad corporal 9,3\%, mientras que los otros grupos no alteraron los valores. Varios estudios científicos han procurado verificar los efectos de programas de entrenamiento de fuerza muscular sobre la fuerza muscular y otras variables en personas ancianas. Estos estudios encontraron mayores aumentos en la fuerza muscular de miembros inferiores que de los superiores variando de $9 \%$ a $227 \%$. Las evidencias sugieren que el entrenamiento de la fuerza muscular puede alterar los efectos negativos de la edad sobre el sistema neuromuscular reduciendo la sarcopenia y ejerciendo mayor impacto en los miembros inferiores que son los que más comprometen la realización de actividades diarias de los ancianos. Recientemente (Evants, 2015) encontró, en una población de 100 ancianos hospitalizados en casas de ancianos, un aumento significante en la fuerza muscular, capacidad funcional y actividad física espontánea después de un programa de entrenamiento de la fuerza muscular de alta intensidad.

En la experiencia del autor con grandes grupos de personas 8.000 un cuestionario simplificado ayuda a detectar los casos de riesgo y con esto no se hace necesaria una evaluación médica para cada individuo para participar de programas de intensidad moderada. Se sabe también que el entrenamiento de resistencia muscular aumenta el metabolismo de reposo, aumenta el gasto calórico, la masa libre de grasa mejora la tolerancia a la glucosa y mejorando la densidad ósea, masa muscular, fuerza, equilibrio y el nivel total de actividad física del individuo, disminuye el de fracturas por osteoporosis en mujeres posmenopáusicas.

Cualquier tipo de paciente puede participar de un programa de entrenamiento de la fuerza, inclusive pacientes con hipertensión arterial, artritis reumatoide u osteoartritis.

Para pacientes con hipertensión es sugerida la realización de un test de levantamiento de peso realizando 3 sets de 8 repeticiones a 80\% de 1RM monitorizando ECG y la presión arterial. Los músculos que deben ser trabajados son aquellos que son utilizados en las actividades diarias y cada repetición debe ser realizada despacio con 2-3 segundos para levantar el peso y 4-6 segundos para bajarlo. 
El entrenamiento de alta intensidad es el que ha mostrado cambios importantes y es considerado como la carga que puede ser levantada 8-12 veces. Deben ser realizados aumentos de carga a cada 2-3 semanas (ya que se observa aumento de $10-15 \%$ en la fuerza por semana en las primeras 8 semanas de programa) y la frecuencia de dos días por semana ya muestra ser benéfica. La orientación más importante para evitar aumentos de presión arterial es inspirar antes de levantar el peso, expirar cuando está siendo levantado e inspirar cuando se baja el peso.

De acuerdo con (Pollock, 2013) el uso de máquinas es preferible al uso de pesos libres en el anciano pues son más seguras, evitan lesiones, permiten incrementos bajos de peso, protegen la columna vertebral y evitan la maniobra de val salva.

Entrenamiento de fuerza muscular y masa ósea:

Existen cada vez más estudios científicos realizados recientemente mostrando los efectos de la actividad física y del entrenamiento de fuerza muscular en la densidad ósea (Pollock, 2013) Los efectos del ejercicio sobre la densidad ósea, son más importantes en las regiones corporales que tienen una mayor cantidad de hueso trabecular, localizado especialmente en los cuerpos vertebrales, (Matzudo, 2011) mientras que los resultados en el hueso cortical, necesitan períodos mayores de entrenamiento de fuerza muscular superior a dos años. Algunos trabajos han mostrado aumento de 2,0\% en la densidad mineral ósea de la columna lumbar y 3,8\% en el contenido mineral óseo del fémur en hombres que realizaron entrenamiento de fuerza muscular durante 16 semanas. Otros resultados muestran que una caminada diaria de cerca de 20 minutos durante un año aumentó $2 \%$ la densidad ósea de 38 mujeres en la pos-menopausia. Datos no conclusivos de (Pollock, 2013), hipotetizan que el entrenamiento de fuerza muscular de alta intensidad ( $80 \%$ de $1 \mathrm{RM})$ realizado tres veces por semana durante 1 año, promueve un aumento de 2 gramos en el contenido total de mineral óseo mientras que las personas que permanecen inactivas reducen cerca de 33 gramas el tejido óseo en el mismo intervalo de tiempo. Los beneficios de estos resultados para el anciano son indiscutibles, porque la carga mecánica provocada por el entrenamiento físico genera un efecto piezoeléctrico en el hueso, generando mayor actividad osteoblástica y aumentando la formación ósea por el aumento en la síntesis de proteínas y de DNA.

De acuerdo con una revisión realizada por (Matzudo, 2011) los estudios científicos realizados hasta el momento indican que el entrenamiento de la fuerza muscular está asociado positivamente con una alta densidad mineral ósea en jóvenes y ancianos, siendo que el efecto es localizado y específico a los locales que están siendo estimulados. A pesar del ejercicio aeróbico ser importante para la masa ósea el entrenamiento de resistencia parece tener un impacto mucho mayor. De esta manera este tipo de entrenamiento es importante para aumentar el pico de masa ósea en las mujeres pre-menopáusicas, para mantener o aumentar masa ósea en las pos-menopaúsicas y para ayudar al anciano frágil a preservar la masa ósea, aumentar masa y fuerza muscular, la agilidad y el equilibrio variables 
fundamentales para la manutención de la capacidad funcional del anciano.

\section{Hidrogimnásia.}

Las principales ventajas de este tipo de actividad en relación a los ejercicios realizados fuera del agua son:

- Disminución de las fuerzas gravitacionales

- Disminución del stress mecánico del sistema músculo-esquelético

- Facilidad para la termorregulación

- Efecto natri urético y diurético

Por estas razones los principales usos de la hidrogimnásia son:

- Limitación de la movilidad articular

- Dificultad en sustentar el peso corporal

- Rehabilitación de lesiones

- Enfermedad renal o hepática

- Dolor lumbar

- Osteoporosis

Las variables que deben ser priorizadas para prescribir actividad física en la tercera edad para mantener la independencia funcional del individuo son en orden de

- Fuerza muscular

- Equilibrio

- Potencia Aeróbica

- Movimientos corporales totales

- Estilo de vida óptima

Una actividad recientemente promovida entre los ancianos es la práctica de Tai Chi Chuan que tiene efectos benéficos mejorando la fuerza muscular, flexibilidad, equilibrio, velocidad de andar y en la potencia aeróbica (Montero, 2012). Pero de la misma forma en que es importante trabajar la fuerza y el condicionamiento cardiovascular del anciano, es fundamental estimular la adopción de un estilo de vida activo. Son estas las tendencias internacionales y la propuesta de la OMS para promover un Envejecimiento Activo. Por esta razón São Paulo lanzó un programa de promoción de salud mediante un estilo de vida activo llamado "Agita São Paulo" que estimula a los adultos mayores a realizar actividades físicas de intensidad moderada, por lo menos 30 minutos al día, la mayor parte de los días de la semana, de preferencia todos, de forma continua o acumulada.

\section{Efectos benéficos de la actividad física en la tercera edad.}

Los principales efectos del ejercicio y la actividad física en la tercera edad pueden ser 
resumidos en (Matzudo, 2011) Efectos antropométricos y neuromusculares: control del peso corporal, disminución de la grasa corporal, aumento de la masa muscular, aumento de la masa magra, aumento de la fuerza muscular, aumento de la densidad ósea, fortalecimiento del tejido conectivo, aumento de la flexibilidad Efectos metabólicos: aumento del volumen sistólico, disminución de la frecuencia cardíaca en reposo y en el trabajo su máximo, aumento de la potencia aeróbica (VO2 máx: 10-30\%), aumento de la ventilación pulmonar, disminución de la presión arterial, mejora del perfil lipídico, mejora de la sensibilidad a la insulina, aumento de la taza metabólica de reposo, Efectos psicológicos: mejora del autoconcepto, mejora de la auto-estima, mejora de la imagen corporal, disminución del stress, ansiedad, tensión muscular e insomnio, disminución del consumo de medicamentos y mejora de las funciones cognitivas y socialización.

Los principales beneficios del entrenamiento de la fuerza muscular en la tercera edad son:

- Mejora de la velocidad de andar

- Mejora del equilibrio

- Aumento del nivel de actividad física espontanea

- Mejora de la auto-eficacia

- Contribución en la manutención y/o aumento de la densidad ósea

- Ayuda en el control de Diabetes, Artritis, Enfermedades cardiovasculares

- Mejora de la ingestión dietética

- Disminución de la depresión

El ejercicio y la actividad física también contribuyen en la prevención de las caídas por diferentes mecanismos:

- Fortalece los músculos de las piernas y columna

- Mejora los reflejos;

- Mejora la sinergia motora de las reacciones posturales;

- Mejora la velocidad de andar;

- Incrementa la flexibilidad;

- Mantiene el peso corporal;

- Mejora la movilidad articular;

- Disminuye el riesgo de enfermedades cardiovasculares.

Además de los beneficios físiológicos de la actividad física en el organismo las evidencias científicas muestran que existen alteraciones en las funciones cognitivas de los individuos que realizan actividad física regular. Estas evidencias sugieren que el proceso cognitivo es más rápido e más eficiente en individuos físicamente activos por mecanismos directos: mejora de la circulación cerebral, alteración en la síntesis y degradación de neurotransmisores; y mecanismos indirectos como: disminución de la presión arterial, disminución de los niveles de LDL en el plasma, disminución de los niveles de triglicéridos e inhibición de la agregación plaquetaria. 


\section{Osteoporosis y actividad física.}

Los estudios científicos realizados en atletas o en individuos físicamente activos demuestran el efecto benéfico del ejercicio en la densidad ósea, la que se observa que estos individuos tienen mayor porcentaje de densidad ósea, que varía de acuerdo con el estudio, en relación a los individuos sedentarios. Los estudios con diferentes programas de actividad física demuestran que el ejercicio tiene efecto benéfico en el sistema esquelético de mujeres con osteoporosis. En relación a las mujeres inactivas, que disminuyen la densidad ósea en el mismo período de tiempo, las mujeres físicamente activas aumentan la densidad ósea en media de 2-5\%. La actividad física, dependiendo de la época de inicio, la duración y la intensidad, contribuye para aumentar el pico de masa ósea, mantener la masa ósea y disminuir la perdida de densidad ósea que ocurre con la edad. La prescripción del ejercicio en estos casos depende de si el objetivo es la prevención, el tratamiento o la rehabilitación.

Para la prevención de la osteoporosis están indicadas las actividades que soportan peso como caminar, correr o trotar. Ya para el paciente con alto risco de osteoporosis o con fracturas, están contraindicadas las actividades que soportan peso. En estos casos son recomendadas actividades como la natación, hidrogimnásia y pedalear en la bicicleta estacionaria. En todos los casos son fundamentales ejercicios para fortalecer la musculatura, principalmente la abdominal y lumbar y evitar siempre ejercicios que requieran flexión de la columna vertebral o torsión del tronco. Por esta razón no están indicados la práctica de tenis, golf o jugar bolos.

Las revisiones más recientes demuestran la necesidad de incluir en cualquier programa de prevención de osteoporosis la realización de actividades de entrenamiento con pesos para la fuerza muscular ya que estas actividades están relacionadas con mayores aumentos de la densidad ósea. Además de estos programas específicos existen evidencias de que el estilo de vida (tiempo del día estando de pie, caminando, subiendo escaleras, cargando peso, cuidando de la casa y del jardín) colabora en la prevención de fracturas por osteoporosis.

\section{Estrategias contra el sedentarismo del adulto mayor.}

El sedentarismo es considerado actualmente el factor de riesgo de muerte más prevalente en todo el mundo (50-70\%). Las evidencias científicas más recientes muestran que sesiones cortas de treinta minutos por día, la mayor parte de los días de la semana, de forma continua o acumulada de 10 a 15 minutos en intensidad moderada, pueden representar el limiar para la población obtener efectos benéficos para la salud. Para esto los científicos sugieren que las personas adopten un estilo de vida activo o sea que incluyan actividades físicas en su día-adía en la casa, en el trabajo o en el tiempo libre. Estas actividades incluyen subir o bajar escaleras, salir a pasear con el perro, cuidar del jardín, lavar el carro, caminatas en ritmo ligero, bailar, pedalear o nadar. Estas nuevas recomendaciones son confirmadas por la Organización Mundial de la Salud (OMS), Consejo Internacional de Ciencias del Deporte y 
Educación Física (ICSSPE), Centro de Control y Prevención de Enfermedades (CDC), Colegio Americano de Medicina Deportiva (ACSM), Federación Internacional de Medicina Deportiva (FIMS) y Asociación Americana de Cardiología. A partir de esta tendencia internacional surgió en São Paulo-Brasil el Programa "Agita São Paulo" con dos objetivos básicos: aumentar el conocimiento de la población sobre los beneficios de la actividad física y aumentar el nivel de actividad física de la población. La propuesta básica consiste en elevar por lo menos en un nivel la actividad física de cada persona, en otras palabras: incentivar el sedentario a ser por lo menos un poco activo, el poco activo a ser regularmente activo; este a ser muy activo y el muy activo a mantenerse en este nivel. "Agita São Paulo" en sus tres principales focos (escolares, trabajadores y ancianos) enfatiza la recomendación mundial de que todo individuo debe: Realizar actividades físicas de intensidad moderada; por lo menos 30 minutos al día; la mayor parte de los días de la semana, de preferencia todos; de forma continua o acumulada. Con esta estrategia fueron ya alcanzadas 33 millones de personas para combatir el principal enemigo de salud: el sedentarismo. Además, en Colombia ya se implantaron varios programas con la misma filosofía y objetivos: el Muévete Bogotá, Risaralda Activa y Muévase Pues. Con todas estas evidencias científicas los investigadores demostraron también que la actividad física aumenta la longevidad. Así entre más activo el individuo más años de vida tendrá independientemente de la edad de inicio de la actividad. En promedio ganamos 2,15 años de vida por los beneficios de la actividad física (Pollock, 2013) Pero tal vez lo más importante no son cuantos años más podemos vivir pero como podemos vivir esa etapa de nuestras vidas con mejor calidad de vida manteniendo la independencia funcional yla autonomía.

\section{Conclusiones.}

- Cierto es que un buen cuidado del cuerpo garantiza una vejez más saludable, para ello se pude tener como referente a cuando hablan acerca de un envejecimiento satisfactorio "en el que se retrasan los cambios involutivos, en el que la alteración de estructuras corporales y la pérdida de las capacidades funcionales es más lenta y que, además, se mantiene libre de problemas y enfermedades incapacitantes o que aumenten el riesgo de muerte"

- La práctica de la actividad física ayuda al mejoramiento del estado fisiológico del cuerpo; siendo el ejercicio un complemento a la prevención de sedentarismo, obesidad, hipertensión, diabetes, estrés, tabaquismo, alcoholismo, drogadicción.

- Por otra parte se observa que esta población, a nivel osteo-muscular, es menos propensa a sufrir alguna lesión, a pesar de enfermedades como la osteoporosis.

- Se puede deducir esto por lo que se aprecia en las clases donde se trabaja con métodos de la fuerza como el auto carga y la utilización de pesos externos como las máquinas y pesos libres, puesto que demuestran una gran fortaleza haciendo 
ejercicios que otras personas, por ejemplo, en el ciclo vital que se encuentra este grupo, no lo harían con tanta facilidad.

- Es entendible que mientras haya un buen cuidado del cuerpo en sus primeros ciclos de vida y en el mismo ciclo de la vejez la calidad de vida será mucho mejor, siendo el objetivo principal aumentar la longevidad.

- Otro beneficio significativo que aporta la actividad física en el adulto mayor lo constituye la dimensión sicológica, en la que algunos autores como Marcos Becerro afirman que "la salud y la capacidad física son importantes elementos del sentimiento de bienestar", por lo tanto, una cuestión importante que debemos considerar es que la población adulta, a través de la práctica del ejercicio, tiende a disminuir sentimientos negativos aumentando así los positivos y su sensación de bienestar.

- Los principales cambios en el comportamiento se dan en lo referido a la autoestima, el auto concepto, la autoeficacia y la imagen corporal. Se puede decir que el auto concepto es el conocimiento que el ser tiene de sí mismo e incluye una percepción sobre su actuar intelectual, social, emocional y físico. Igualmente la autoestima es la apreciación positiva o negativa que el sujeto tiene de sí mismo.

- En este orden de ideas se entrelaza la imagen corporal como un importante referente del trabajo o práctica del ejercicio. Becerro hace mención de una investigación que se realizó hace más de dos décadas, donde se encontró que "después de realizar un programa de ejercicio durante catorce semanas, se observó que la imagen corporal cambiaba en aquellos que hacían más ejercicio”.

- Esto es, los sujetos que entrenaban más, con mayor frecuencia e intensidad. Finalmente, otro importante componente del bienestar es la auto- eficacia; vista desde el componente físico creencia que tiene un sujeto acerca de su propia capacidad de ejecutar tareas específicas. Siendo entonces la auto-eficacia la percepción del individuo sobre la capacidad que tiene para realizar una actividad. En este punto es claro el concepto de vejez y la importancia de realizar actividad física con el fin de prolongar el estado de salud óptimo.

- Al igual que ocurre con otras capacidades condicionales, la fuerza se ve beneficiada por la práctica de la actividad física; dando como resultado un mejor desempeño en las actividades cotidianas (levantar, empujar y sostener pesos), sostener posiciones prolongadas así como una adecuada fuerza en las piernas puede prevenir una caída por diversas razones, como corrección de la pérdida momentánea del equilibrio con el objeto de prevenir caídas catastróficas y la fuerza de miembros superiores puede reducir la cantidad de lesiones que resultan de una caída por falta de fuerza para estabilizar las articulaciones durante la caída. 


\section{Referencias bibliográficas.}

Andrade, (2014). Niveles de fuerza en el adulto mayor .Sensación.

Evants, (2015). Physical activity and sport in elderly. Sport. Matzudo, d. S. (2011).

Actividad fisica pasaporte para la salud .

[rev. Med. Clin. Condes - 2012; 23(3) 209-217].

Montero, m. F. (2012). Ejercicios de taichí para la estabilidad de la tensión arterial . Ef deportes.

Pollock, f. (2013). Benefits of exercise of force in the elderly.

Axion.

Raso, m. (2014). Ejercicios específicos para el adulto mayor.

Scielo.

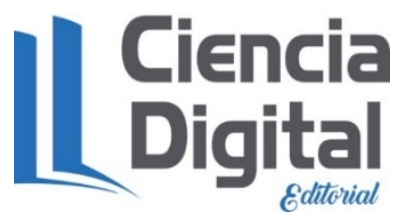




\section{Para citar el artículo indexado}

Flores, M. C., Carrasco Coca, O. R., Ochoa Sangurima, V. L., \& Ríos Bayas, R. S. (2020). Desarrollo de la fuerza en el adulto mayor a través de la hidrogimnásia en la calidad de vida. AlfaPublicaciones, 2(3), 55-66. https://doi.org/10.33262/ap.v2i3.35

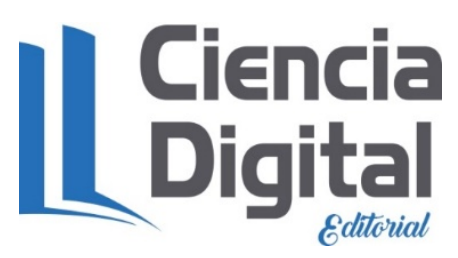

El artículo que se publica es de exclusiva responsabilidad de los autores y no necesariamente reflejan el pensamiento de la Revista Alpha Publicaciones.

El artículo queda en propiedad de la revista y, por tanto, su publicación parcial y/o total en otro medio tiene que ser autorizado por el director de la Revista Alpha Publicaciones.
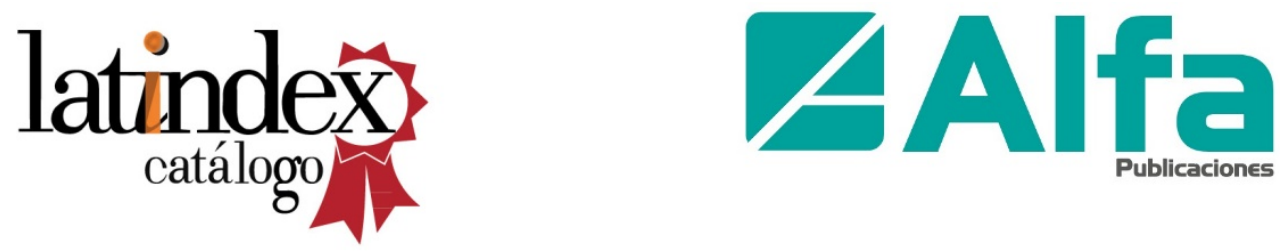